\title{
Influencia de las Redes Sociales como herramienta de interacción en la educación.
}

\section{Influence of Social Networks as an interaction tool in education.}

\author{
Efraín Velasteguí López. ${ }^{1}$
}

\section{Resumen.}

La influencia de las redes sociales se ha convertido en herramientas indispensables para jóvenes estudiantes cuyas rutinas diarias están siempre relacionadas con el entorno social que les rodea. En lo antes descrito y para reforzar lo anterior, también hemos de tener en cuenta que el lugar de procedencia del estudiante no le condiciona para hacer uso de las redes sociales, puesto que hay una amplia mayoría que hace uso de las mismas, sobre todo si hablamos de estudiantes mayores de 18 años. Sin embargo, si hay que matizar que el país de residencia influye el uso de una u otra red social.

En ese contexto, existen diferentes recursos didácticos aptos para la introducción y adquisición de conocimientos, habilidades, actitudes, etc. Se muestra una reflexión sobre los recursos didácticos que favorecen el aprendizaje de nuevas competencias y una propuesta acerca de los nuevos roles que deben adoptar docentes y estudiantes ante las nuevas tecnologías, haciendo énfasis en el proceso de obtención, tratamiento, síntesis y comunicación de la información, además del análisis de las circunstancias que rodean el uso de dichas tecnologías. La influencia de las redes sociales nos permite el aprendizaje colaborativo e involucra espacios de intercambio de información que fomenta la cooperación, y además nos permite tener más conocimientos sobre de las nuevas tecnologías y así percibir y procesar la información en el ámbito de la educación. Permite comprender la relación entre el concepto de estilos de aprendizaje que distintos autores han construido y lo que los estudiantes desarrollan al utilizar herramientas como las redes sociales.

Palabras claves: redes sociales, internet, tecnología, educación. Herramienta de aprendizaje, comunicación.

\footnotetext{
${ }^{1}$ Ciencia digital, Ambato, Ecuador, luisefrainvelastegui@ cienciadigital.org
} 


\section{Abstract.}

The influence of social networks has become indispensable tools for young students whose daily routines are always related to the social environment that surrounds them. In the above described and to reinforce the above, we must also bear in mind that the place of origin of the student does not condition him to make use of social networks, since there is a large majority that makes use of them, especially if We are talking about students over 18 years of age. However, if it is necessary to clarify that the country of residence influences the use of one or another social network.

In this context, there are different didactic resources suitable for the introduction and acquisition of knowledge, skills, attitudes, etc. It shows a reflection on the didactic resources that favor the learning of new competences and a proposal about the new roles that teachers and students must adopt before the new technologies, emphasizing the process of obtaining, processing, synthesis and communication of information, in addition to the analysis of the circumstances surrounding the use of these technologies. The influence of social networks allows collaborative learning and involves spaces for information exchange that encourages cooperation, and also allows us to have more knowledge about new technologies and thus perceive and process information in the field of education. It allows us to understand the relationship between the concept of learning styles that different authors have constructed and what students develop when using tools such as social networks.

Keywords: social networks, internet, technology, education. Learning tool, communication.

\section{Introducción}

\section{Influencia de las redes sociales como medio en la educación}

Imagen N.1: Las redes sociales

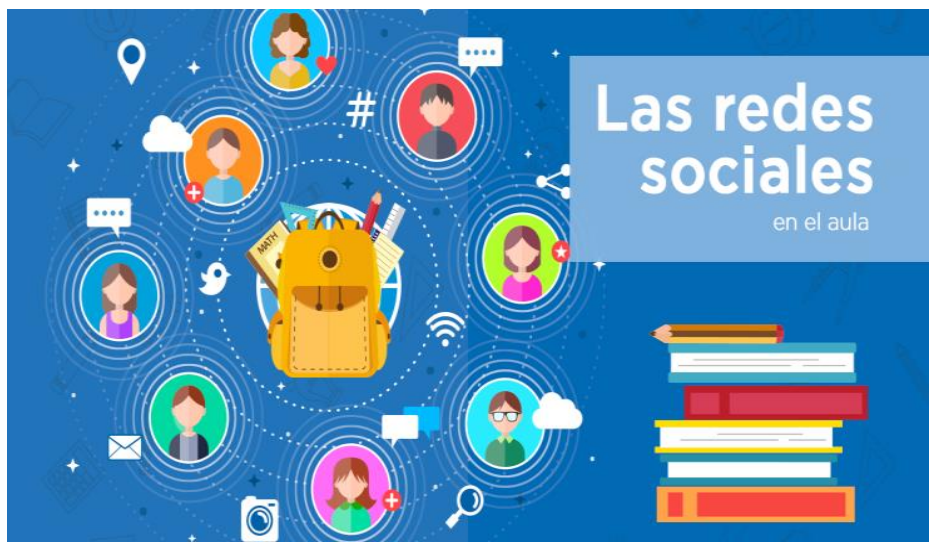

Fuente: https://www.redem.org/educacion-3-0-usos-de-las-redes-sociales-en-elaula-ventajas-y-desventajas/

Elaborado por: María Lozano

Influencia de las redes sociales como medio de la educación según la investigación obtenida de (Hoyos, 2007; Gómez, 2012 y Barabasi, 2002) afirman que se han 
convertido en protagonistas de su propia formación supone que se realicen los cambios suficientes para que dicho cambio no resulte traumático para ambas partes implicadas en el proceso. Ante la convergencia, docentes y estudiantes deben ser conscientes de los nuevos papeles que deben asumir en el proceso de aprendizaje. Hoy en día es imprescindible el conocimiento del uso del internet para poder optar a un puesto de trabajo, independientemente de las tareas que se tengan que desempeñar, las ideas deben contemplarse en la formación universitaria que reciben los estudiantes que se matriculan en cualquier título oficial con el fin de que estén bien preparados para su futura vida laboral.

Las influencias de las redes sociales según la investigación realizada de distintos autores dicen que son una herramienta muy importante ya que en la cual nos sirve de mucho apoyo en la sociedad atreves de las redes sociales nos podemos comunicarnos a distintos lugares de país con varias personas que se encuentran fuera del país, juega un papel importante ya que se pueden realizar trabajos, que sean enviados por los docentes o realizarlos mediante online.

Las redes sociales en la educación es un cambio en el cual tienen la posibilidad de que vean y aprendan cosas nuevas con el avance de las nuevas tecnologías ya que cada día que pasan salen nuevas cosas en la tecnología, para que los cambios no se queden en una mera alteración del soporte habrá que producirse una autentica revisión del uso que actualmente se les está dando.

Ya que son un medio muy importante en la cual se pueden obtener distintos trabajos y consultas ya que son materiales multimedia disponibles son de muy baja calidad, reproduciendo modelos de enseñanza-aprendizaje de tipo conductista y que generan escasos retos intelectuales a quienes nos manejan, por tanto, el progreso tecnológico queda reducido a la incorporación de soportes novedosos mientras que la práctica didáctica no solo mejora, sino que podría, incluso retroceder. Como también se dice que los materiales que se necesitan para realizar alguna investigación se necesita tener un internet con mucha potencia para poder realizar el trabajo necesario sin tener ninguna interrupción.

Como también es un avance en la manera de educar a los estudiantes ya que ahora se realizar los trabajos por medio del internet ya sean que se envíen por correo electrónico, ya que anteriormente se lo enviaban trabajos que sean realizados a mano y sean presentados al siguiente día.

En las estadísticas actuales, tanto a nivel mundial, como en nuestro país, es notorio que está creciendo de manera importante el uso de las redes sociales, por lo cual, es conveniente considéralas como espacios factibles para hacer llegar la información educativa a los estudiantes que, una vez conectados. Pueden aprovechar su tiempo para consultar contenidos educativos e interactuar como profesores y otros alumnos.

Gráfico N.1: Redes Sociales 2017 usuarios activos mensuales 


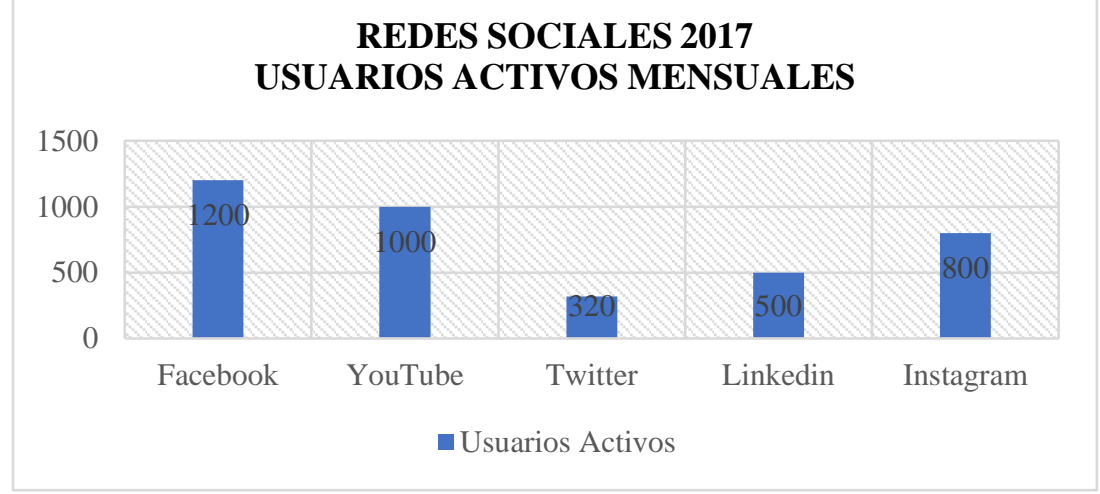

Fuente: Investigación

Elaborado por: Jessica Doicela

La influencia de las redes sociales según la investigación realizada de (Aguades, 2008; Ramírez, 2008 y Esteruelas, 2012) afirma que estos contenidos de hoy en día ya se encuentran disponibles diversos sitios y servicios web que facilitan la generación de redes sociales privadas y públicas con fines específicos, las cuales pueden aprovecharse por los docentes interesados. Por otro lado, si el docente no tiene conocimientos teóricos o no está interesado en generar una red social propia, es posible emplear las redes existentes restringiéndolas a sus fines académicos, cuidando siempre la seguridad en la identidad, tanto de los usuarios, como le da información que se publica.

Las redes sociales permiten a los usuarios realizar publicación de distintas informaciones que se deben ser publicadas en distintas páginas web, el aprendizaje autónomo, el trabajo en equipo, la comunicación, la realimentación, el acceso a otras redes afines y el contacto con otros expertos, entre otros elementos. Todo ello, tanto entre estudiantes en general, como entre en binomio estudiante y docente; lo cual facilita el aprendizaje constructivista y el aprendizaje colaborativo.

Permite el aprendizaje autónomo a los estudiantes y facilita la realización de tareas ya que se podrían hacer vía online y no sería necesario asistir a las universidades a presentar ningún tipo de trabajo y serian realizados en sus propios hogares, como también se debe tener en cuenta cuando se usan las redes sociales se deben usar de manera adecuada.

Parece claro que, hoy por hoy las nuevas tecnologías se han convertido en una herramienta insustituible e indiscutible valor y efectividad en el manejo de la información con propósitos didácticos. Ya no es suficiente que los docentes sean buenos manejadores de libros. Las fuentes de información y los mecanismos para distribuirla se han informatizado y resulta fácil poder concebir un proceso didáctico en la universidad sin considerar esta competencia. La incorporación de las tecnologías de Información y Telecomunicaciones (TICs) a la enseñanza requiere un cierto nivel de competencia técnica, no sabe ninguna duda de que nuevas tecnologías de la información y la comunicación están llamadas a alterar profundamente la docencia universitaria.

Según la investigación realizada de distintos autores mi opinión es que se debe tenerse en cuenta la inclusión de las redes sociales dentro del estudio académico al beneficiar la comunicación académica y la interacción entre los docentes y los estudiantes, y entre los mismos estudiantes, pero debe tenerse en cuenta una adecuada orientación en los aspectos 
de seguridad de la información y el correcto uso de las herramientas de apoyo que pueden brindar en campo de estudio universitario.

Las redes sociales según las investigaciones realizadas de (Ramírez, 2008; Rodríguez, 2012 y Dueñas, 2008) afirman que la influencia de las redes sociales nos brinda diversidad en el uso de distintos formatos como textuales, audiovisuales, hipervínculos y gráficos, que incluyan plataformas de comunicación donde aportan documentación electrónica o enlaces al instantes, y que faciliten la conformación de grupos de trabajo de investigación bajo tutoría e incluso de grupos de trabajo entre los mismos estudiantes. Facebook pueden ser un vínculo importante de interacción dentro del desarrollo del estudio universitario para facilitar el desarrollo de actividades académicas entre los docentes y los estudiantes y entre ellos mismos por lo que pueden utilizarse los grupos cerrados para compartir información y desarrollo de asignaciones e investigación.

Las redes sociales se han dado un gran impacto en la actualidad ya que atreves de las cuales se pueden realizar trabajos vía online, y poderse comunicarnos entre compañeros sin necesidad de reunirse para realizar algún trabajo que sea de realizar en grupo de trabajo si no ser realizados mediante las páginas web como correos electrónicos, Facebook.

Como también se debe existir la debida orientación de seguridad para evitar cualquier tipo de problemática que se pueda ocurrir durante la utilización de distintas redes sociales, la cual se pueden realizar mediante la información personal o institucional. Ya que la inversión de tiempo de los estudiantes en las rede sociales es muy elevada, se debe incluir dentro de la plataforma didáctica la respectiva interacción en estas que puedan brindar un balance entre las horas de estudio y las horas de desvinculación de sus hábitos de estudio. La visión de las redes sociales es indagar al mayor número de miembros posibles, esto gracias al ofrecimiento de diversas herramientas que garantizan la fidelidad de los usuarios. Ente estas herramientas, correo electrónico, diseminación de la información personal, compartir fotos, videos y mensajería instantánea en mensajes de texto.

En mi criterio será que las redes sociales son un medio de entretenimiento, en la cual se ve relacionada con la sociedad también es un medio en la cual se pueden encontrarse o hacerse amigos con personas que talvez no conozcamos y no son utilizan con un fin lucrativo. creo que la red social debería de haber algunas restricciones al respecto con distintas páginas sobre todo y principalmente con menos de edad. Como también se puede decir que las redes sociales son un tipo de comunicación, información.

\section{Redes sociales como herramienta de aprendizaje}

Imagen N.2: Como utilizar los medios sociales como una herramienta de aprendizaje 


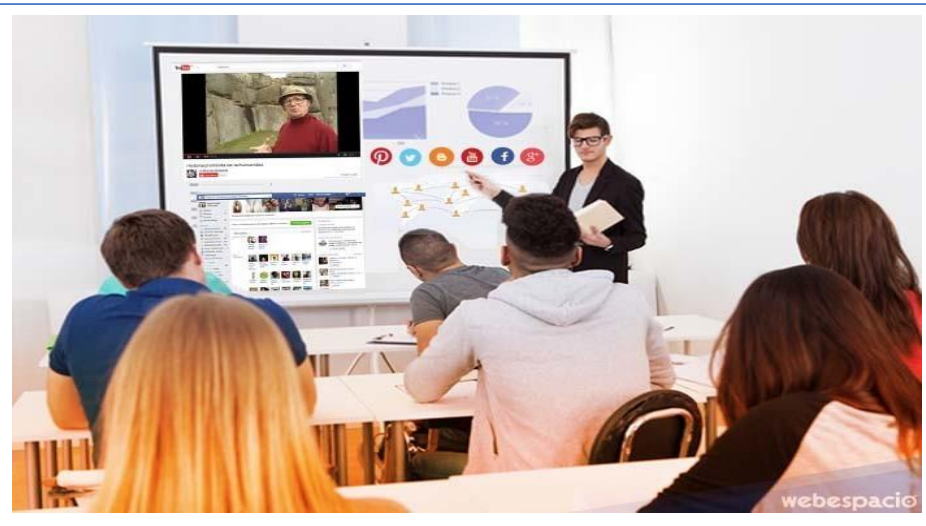

Fuente: https:www.webespacio.com/utilizar-medios-sociales-herramientaaprendizaje-aula/

Elaborado por: Junny Villugas

Las redes sociales según la investigación realizada de (Body, 2007; Allison, 2007) afirman que es un servicio de web que permite a los individuos construir un perfil público o semi-publico dentro de un sistema delimitado, articular una lista de contactos y ver cruzar sus lista de conexiones y las hechas por otros dentro del sistema que es medio útil para compartir todo tipo de datos de información y múltiples formatos: audios, testo y videos, y con muchas menos limitaciones que el correo electrónico, cuya capacidad es mucho más limitada.

Estas nuevas herramientas brindan una amplia capacidad de comunicación que facilita el fluido de información de toda clase, ya que, si bien es un principio se utilizaban para publicar, esencialmente, reflexiones o viviendas de los usuarios, hoy en día casi todos los informáticos y grandes medos de comunicación cuentan con perfiles en esta red social.

A sido un buen avance en las tecnologías ya que atreves de ellas se va obteniendo un poco más de conocimiento y así poder intercambiar información, y como también para estar enterados de la información que acontece en el ecuador y en el mundo, como también en la actualidad han cambiado vidas, como en el ámbito laboral,

Como también se pueden realizar publicaciones en la página web y que las publicaciones realizadas puede ver en distintos lugares del mundo ya asean anuncios de trabajo, ofertas que tenga la empresa, la cual han ayudado a mantener en contacto a una enorme red mundial que conecta a organizaciones, comunidades y personas; son el más fácil, rápido y nuevo medio de poder transmitir o recibir información, lo mejor es que cualquier persona le puede utilizar, inclusive de pequeñas y grandes empresas, han aprovechado estos medios para llegar a nuevos clientes.

Los seres humanos siempre hemos experimento la necesidad natural y prioridad $\mathrm{u}$ mantenernos relaciones entre sí, como familia como amigos y en el entorno laboral, como parte activa en la sociedad en constante desarrollo teniendo en la tecnología una opción importante para el alcance y consolidación de tal finalidad, ya que esta nos presta una diversidad de diferentes modalidades e innovaciones creativas que nos facilitan esa comunicación, sin importar lo lejos o separados que estemos unos de otros.

Estas redes sirven para vender, mostrar los productos y ganar popularidad en un negocio, se utiliza: 
Tabla N.1: Redes sociales más utilizadas por las empresas para ofrecer sus productos

\begin{tabular}{|c|}
\hline EDES SOCIALES MÁS UTILIZADAS POR LAS EMPRESAS \\
\hline Facebook \\
\hline Instagram \\
\hline Páginas web \\
\hline
\end{tabular}

Fuente: Investigación

Elaborado por: Jessica Doicela

A medida que pasa el tiempo observamos que, a la hora de comunicarse con un cliente, los vendedores prefieren utilizar una red social con el fin de comunicarse, en vez de hacerlo en persona. Esto le ha dado muy buenos resultados a la hora de tener una conversación entre vendedor y comprador, para las personas que tienen negocios pequeños.

En mi opinión es un medio en el cual podemos comunicarnos con personas y familiares que no se encuentran juntos a través de las redes sociales siempre y cuando utilizando de una buena forma. En la cual nos permite comunicarnos entre amigos y familiares, enviar trabajos, realizar consultas.

Tabla N.2: Mayor uso de las redes sociales

\begin{tabular}{|l|c|c|}
\hline \multicolumn{1}{|c|}{ Uso de la red social } & Frecuencia & Porcentaje \% \\
\hline Enviar y recibir mensajes a familiares y amigos & 54 & 32.34 \\
\hline Intercambiar información y conocimiento & 29 & 17.37 \\
\hline Motivo de estudio o profesional & 24 & 14,37 \\
\hline Intercambiar multimedia & 21 & 12,57 \\
\hline Participar en debates & 21 & 12,57 \\
\hline Diversión y ocio & 18 & 10,78 \\
\hline TOTAL & 167 & 100 \\
\hline
\end{tabular}

Fuente: Investigación

Elaborado por: Jessica Doicela

Gráfico N.2: Mayor uso de las redes sociales 


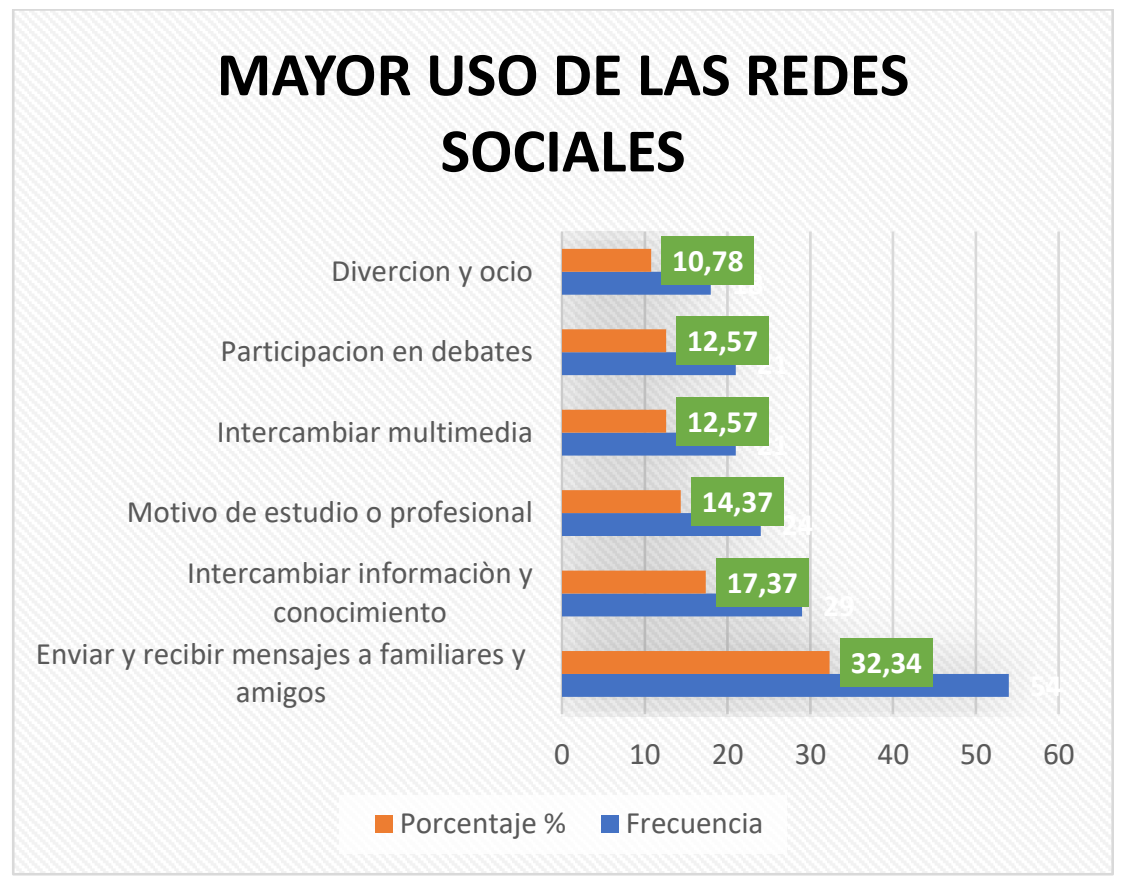

Fuente: Investigación

Elaborado por: Jessica Doicela

\section{Ventajas y desventajas del uso de las nuevas tecnologías}

Las redes sociales según la investigación realizada de varios autores (Adell, 1997; Dueñas, 2008 y Body, 2007) afirman que las redes sociales en la educación son precisamente una de las formas que el internet brinda para comunicarnos con las personas. Se enmarcan en la denominación de web 2.0., y supone una forma interacción social que se basa en el intercambio interactivo y dinámico de distinta información entre diferentes personas, grupos no institucionales. La educación ha sido una de las disciplinas que más se está beneficiando de este tipo de redes sociales, las redes sociales han favorecido especialmente el trabajo en grupo y la colaboración entre pares, en un espacio ideal para compartir conocimientos que resulten atractivos y motivadores para el estudiante.

Como también las redes sociales según los avances de las tecnologías son un medio importante en la cual se comparten información muy relevante para distintas fuentes de trabajos y así fortalecer conocimientos en distintas personas. Según cómo avanza la tecnología de hoy en día nos permite que las clases de hoy sean aún más motivadoras para el desarrollo de nuestras preparaciones.

Tabla N.3: Las redes sociales tienen ventajas desventajas en común:

\begin{tabular}{|c|c|}
\hline VENTAJAS & DESVENTAJAS \\
\hline $\begin{array}{l}\text { Minimizar las necesidades de formación } \\
\text { porque todos usan el mismo recurso. }\end{array}$ & $\begin{array}{c}\text { Perdida de la privacidad de la } \\
\text { información. }\end{array}$ \\
\hline $\begin{array}{l}\text { Favorece la comunicación con los } \\
\text { alumnos de manera bidireccional, pues }\end{array}$ & $\begin{array}{l}\text { Al ser una comunidad abierta todas las } \\
\text { personas tienen acceso a todo tipo de }\end{array}$ \\
\hline
\end{tabular}




\begin{tabular}{|c|c|}
\hline $\begin{array}{c}\text { el profesorado y el alumnado se } \\
\text { encuentran en el mismo espacio. }\end{array}$ & $\begin{array}{c}\text { información que se edite o que sea } \\
\text { subida. }\end{array}$ \\
\hline $\begin{array}{c}\text { Posee un carácter generalista que } \\
\text { posibilita el uso universal de las } \\
\text { mismas. }\end{array}$ & $\begin{array}{c}\text { La saturación de usuarios puede } \\
\text { convertirse en un momento determinado } \\
\text { en una gran desventaja, ya que esto } \\
\text { permite un cuello en la botella en un } \\
\text { mundo de lluvia de ideas desde diferentes } \\
\text { horizontes y de diversos usuarios. }\end{array}$ \\
\hline $\begin{array}{c}\text { Nos brindan información importante a } \\
\text { través de los sitios de la red, desde } \\
\text { asuntos sin importancias hasta los más } \\
\text { relevantes. }\end{array}$ & $\begin{array}{c}\text { No todas las redes sociales son de la } \\
\text { misma naturaleza ni ofrecen las mismas } \\
\text { herramientas educativas, académicas y } \\
\text { profesionales. }\end{array}$ \\
\hline $\begin{array}{c}\text { Muchas empresas cuentan con entornos } \\
\text { sociales por los cuales comunican las } \\
\text { oportunidades laborales. }\end{array}$ & $\begin{array}{c}\text { Querer incorporar la web 2.0 en la } \\
\text { educación superior, aunque otros rescatan } \\
\text { estomo ahorro de tiempo y dinero al } \\
\text { momento de buscar o difundir la } \\
\text { información. }\end{array}$ \\
\hline $\begin{array}{c}\text { Facilita el estudio en nuestra } \\
\text { competencia. }\end{array}$ & Requiere del personal cuantificado \\
\hline $\begin{array}{c}\text { Son una excelente herramienta de } \\
\text { networking. }\end{array}$ & No son gratuitas. \\
\hline
\end{tabular}

Fuente: Investigación

Autor: Jessica Doicela

Sin embargo, no podemos referirnos a las redes sociales en educación sin tener en cuenta tres puntos de vista que nos indican hasta qué punto son esenciales en la actualidad.

$>$ Aprender con las redes sociales. - el hecho de que los jóvenes utilicen tanto las redes sociales, nos da pie a que intentamos sacar el máximo partido de ellas.

Aprender a través de las redes sociales. - se relacionan con los procesos de aprendizaje informales, en donde los estudiantes pueden aprender de forma autónoma.

$>$ Aprender a vivir en un mundo de redes sociales. - es necesario concienciar a los nuevos usuarios que son redes sociales y para que las pueden utilizar.

Las redes sociales en la educación permiten establecer una comunicación óptima entre los estudiantes y docentes, es necesario que se den algunas condiciones idóneas:

$>$ Es necesario disponer de recursos tecnológicos que den respuesta a las necesidades de los docentes, así como el disponer de una tecnología adecuada.

$>$ Es imprescindible ofrecer recursos formativos que aseguren una correcta adquisición de competencias por parte del docente. 
A través de las redes sociales educativas los usuarios comparten ideas, conocimientos sobre una determinada disciplina, muestran sus trabajos y platean preguntas que les garantizan una atención más individualizada, porque el estudiante tiene un papel activo en su aprendizaje. El docente debe ser un guía que facilite que el alumno poco a poco pueda ser capaz de aprender cada vez de manera más autónoma. Es necesario que los docentes se planteen como incluir las redes sociales en las practicas diarias, pues son medios que pueden facilitar la innovación educativa.

Estas tecnologías permiten a los alumnos interactuar con los contenidos y comunicarse a través de diferentes medios, seleccionarlos, crearlos y compartirlos. Hacer uso de las redes sociales permitirá al docente:

> Fomentar el trabajo colaborativo en su asignatura.

$>$ Abrir la asignatura más allá de las paredes del aula.

$>$ Acceder al conocimiento al instante y seguir actualizado.

$>$ Crear una red de contactos docentes y profesionales, a la vez que acerca a los alumnos de su asignatura a la realidad profesional.

$>$ Desarrollar competencias informacionales que permitan gestionar la gran cantidad de información que circula en la red.

$>$ Crear o mejorar una reputación y visibilidad en la red.

$>$ Compartir o reflexionar los trabajos generados y experiencias.

Las redes sociales según las investigación de (Uribe, 2018; Rodríguez, 2008 y Pineda, 2009) afirma que ha sido abordado como temática de gran interés, teniendo en cuenta las constantes transformaciones sociales y de modernización que de alguna forma inciden en el debilitamiento y deterioro de los lazos sociales; repercutiendo en diversas problemáticas que afectan la salud, el bienestar y calidad de vida de las personas que integran dichas sociedades, por eso es necesario destacar la importancia que tiene la ayuda que reciben los individuos de los sistemas sociales informales (familia, amigos, vecinos) sobre su salud, sin afirmar que siempre sean beneficiosos. Otro aspecto que destaca la importancia del apoyo social es el concepto de salud mental que se ha trabajado durante los últimos años, ya que ha tenido en cuenta los factores psicosociales como un factor determinante en la salud de los individuos de la sociedad.

Las redes sociales según la investigación realizada me parecen de gran interés, pero también hay que tener en cuenta muchos factores que se deben tomar en cuenta, como bien es cierto las redes sociales en la educación superior nos permite obtener mucho más conocimiento en lo que ocurre cada día que pasa va cambiando la tecnología.

Sin lugar a dudas, las herramientas online pueden enriquecer el proceso de aprendizaje y también mejorar, perfeccionar la enseñanza. Por ello, las oportunidades de las redes sociales al sistema educativo son de un gran valor.

Compartir no solo conocimientos, sino también experiencias

$>$ Posibilidad de que los profesores ayuden a los alumnos a valerse por sí mismo, por ejemplo, a la hora de buscar información relevante para ellos, de tal manera que puedan aprender "haciendo cosas" 
Fomento del trabajo cooperativo entre los propios alumnos

El objetivo es poner en contacto la comunidad académica, permitiendo "ejercer el rol de alumno o profesor, compartiendo sus conocimientos, sus trabajos o su experiencia con los demás miembros de la comunidad", y EDU 2.0 desarrollada por Graham Glass, que "pretender dar una respuesta a las necesidades y metodologías educativas actuales, ofreciendo a los docentes y profesionales del sector una plataforma gratuita con más 10.000 recursos educativos aportados por otros docentes de todo el mundo", son ejemplos de redes académicas.

Gráfico N.3. Consumo y uso de las TICS en el Ecuador

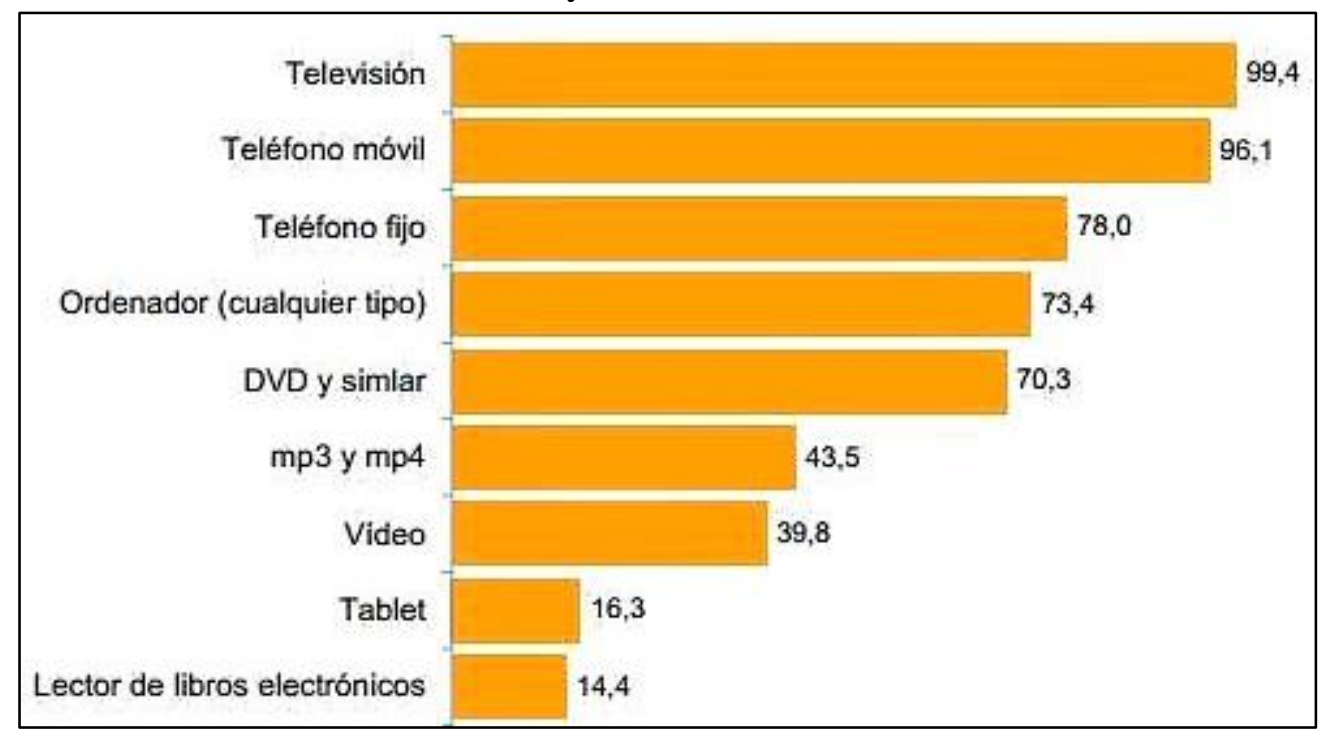

Fuente: http://inforycomun.blogspot.com/2014/11/consumo-y-uso-de-las-ticsen-el-ecuador.html

\section{Elaborado por: Gisela Celín}

Las tecnologías de la información y las Comunicación TICS, en nuestro país ha evidenciado un peligroso retraso en el uso de la tecnología y el internet. Por lo que la mayoría de la gente no cuenta con acceso a estos recursos tecnológicos.

Según el Instituto Ecuatoriano de Estadística y Censos (INEC), el 35\% de la población de Ecuador ha utilizado el Internet en los últimos años, por lo que las TICS son herramientas fundamentales relacionadas a los sistemas informáticos para la transmisión e intercambio de información.

Gráfico N.4. Consumo y uso de las TICS en el Ecuador 


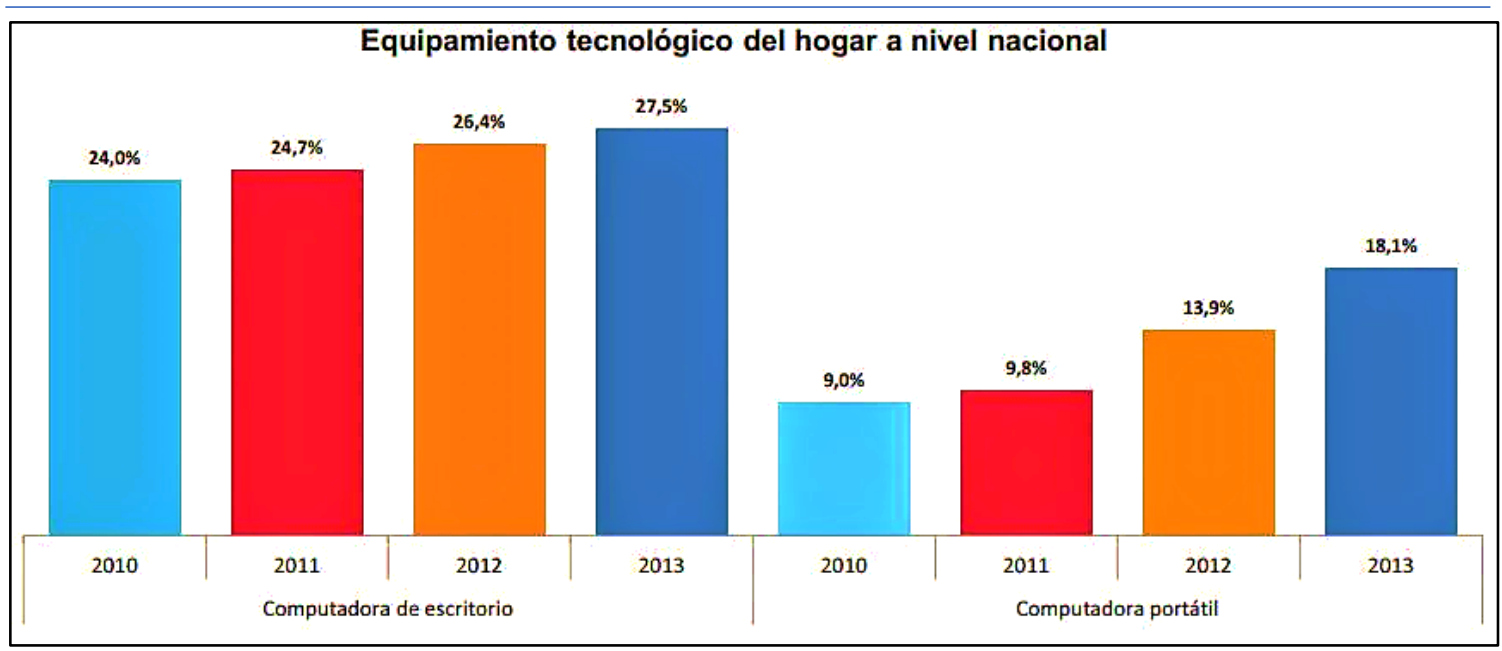

Fuente: http://inforycomun.blogspot.com/2014/11/consumo-y-uso-de-las-ticsen-el-ecuador.html

Elaborado por: Gisela Celín

En Ecuador sigue un constante avance en cuanto al uso y acceso a todo equipo tecnológico: portátil o de escritorio. Se considera que al menos el 18.1\% de los hogares disponen de un computador portátil mientras que el $27 \%$ tiene un computador de escritorio.

Gráfico N.5. Redes Sociales más usadas en el año 2018

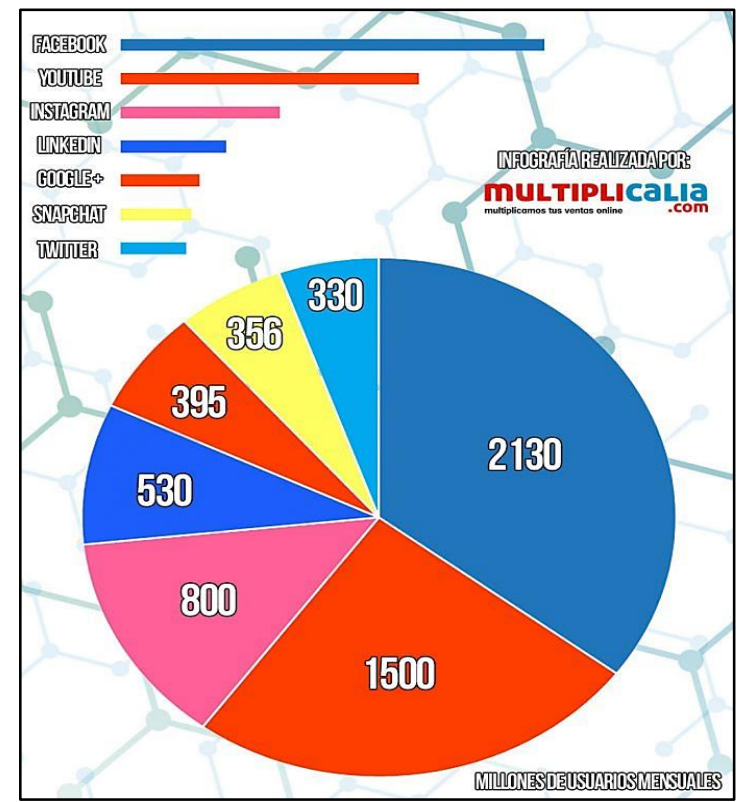

Fuente: https://www.multiplicalia.com/redes-sociales-mas-usadas-en-2018/ Elaborado por: Hugo Giulietti

Siguiendo en su línea, Facebook aumenta casi otros 300 millones de usuarios para llegar hasta los 2.130 millones de MAU. Una barbaridad, si además tenemos en cuenta que en 
todo el planeta somos, ahora mismo, 7.603 millones de humanos. Es decir, 1 de cada 4 humanos se conecta a Facebook cada mes. Impresionante.

Gráfico N.6. Hogares que tiene teléfono fijo y celular a nivel nacional.

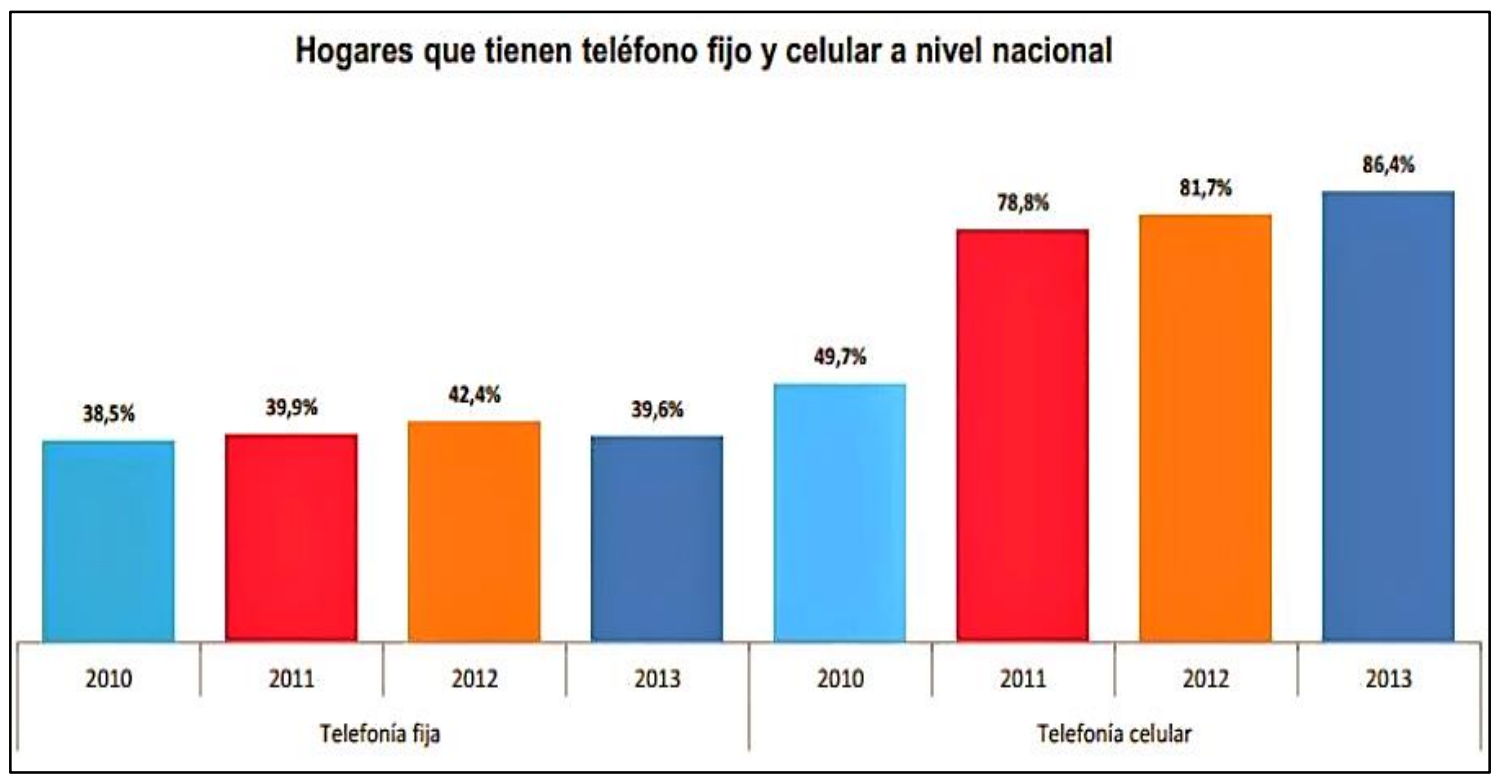

Fuente: http://inforycomun.blogspot.com/2014/11/consumo-y-uso-de-las-ticsen-el-ecuador.html

Elaborado por: Gisela Celín

En cuanto a telefonía móvil y telefonía fija según el INEC, un 86,4\% de los hogares disponen de un celular que a comparación del último censo realizado en el 2010 existe un constate aumento de 36,7 puntos.

Existe un creciente alto en la telefonía móvil alcanzando un 86,4\% a comparación de la telefonía fija se observa una baja utilización de esa telefonía alcanzando un 39,6\% es meno de la mitad de usuarios. Relativamente es evidente que el uso y acceso del abonado de preferencia en telefonía móvil.

Gráfico N.7: Uso de redes sociales por generación a nivel Mundial 


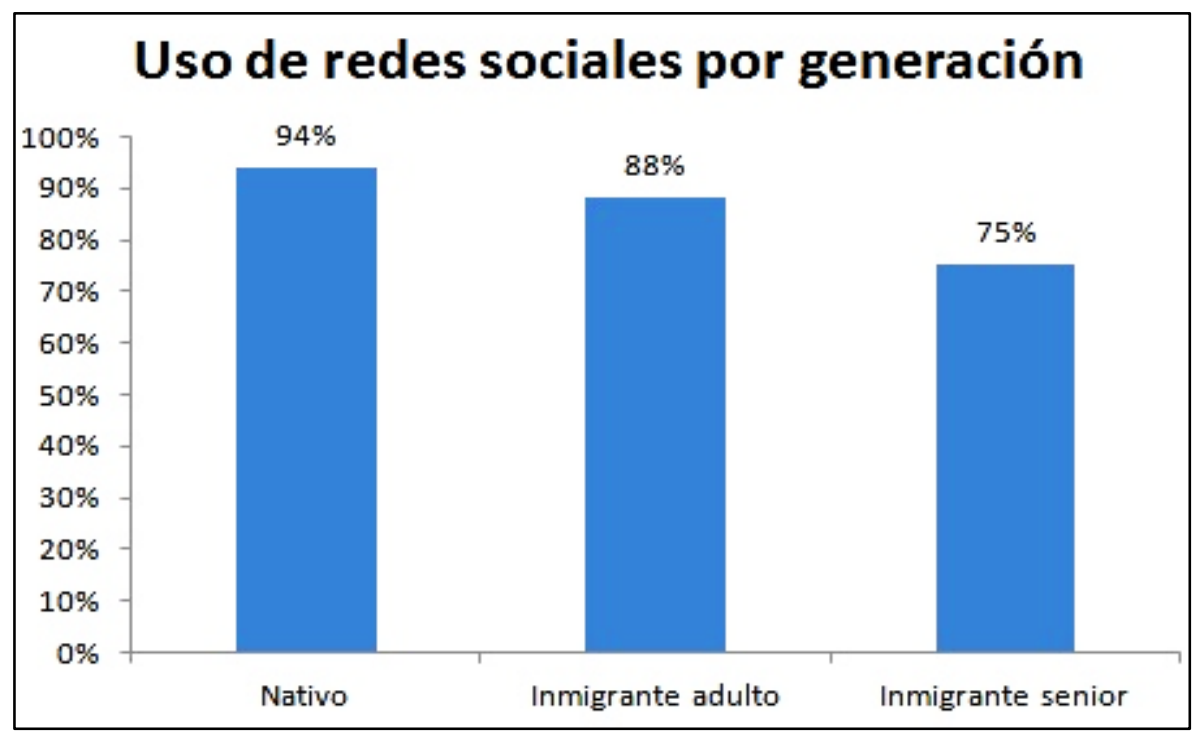

Fuente: http://www.comentariosblog.com.ar/2012/08/24/fenomeno-social/ Elaborado por: Carrier y Asoc.

En la investigación realizada podemos observar que el uso de las redes sociales a nivel mundial, en la nacionalidad nativo es del $94 \%$, inmigrante adulto es del $88 \%$ y inmigrante senior es de $75 \%$.

Gráfico N.8: Evolución de las redes sociales

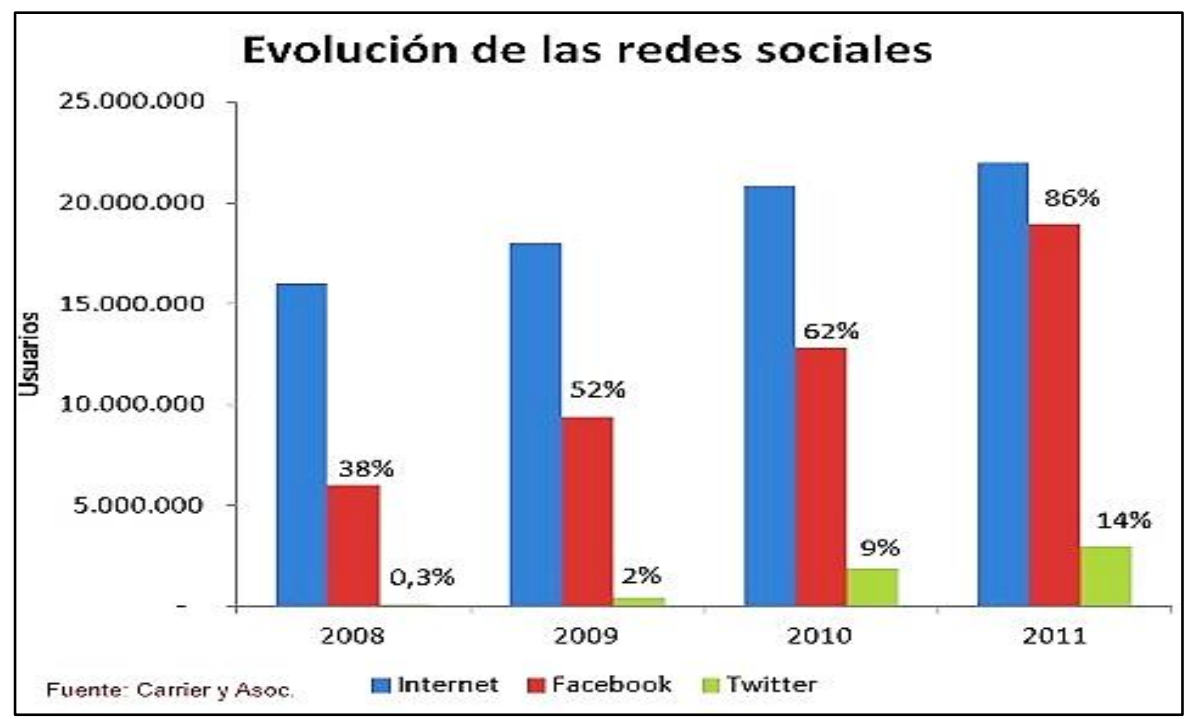

Fuente: http://www.comentariosblog.com.ar/2012/08/24/fenomeno-social/ Elaborado por: Carrier y Asoc.

En la investigación realizada podemos observar que la evolución de las redes sociales en el 2008 es de $38 \%$, en el 2009 es del $52 \%$, en el 2010 es del $62 \%$ y en el 2011 es del $86 \%$. 


\section{Conclusiones}

$>$ Considero que las redes sociales significan un gran avance para la tecnología y gracias a ellas podemos conocer personas; hacer parte de comunidades de conocimientos o de aficiones, pero a pesar de múltiples ventajas que ofrece también ha cambiado enormemente la forma de interactuar con los demás y es así donde juegan un papel fundamental los familiares.

- Las redes sociales son páginas web distintas exclusivamente para usuarios que quieran interactuar con otras personas, desde cualquier parte del mundo siempre y cuando se dispongan de una computadora y que tenga servicio de internet o un dispositivo con tecnología de redes sociales incorporados.

$>$ Como bien es cierto las redes sociales se pueden convertir en una herramienta didáctica- tecnológica en la que se puede fomentar el aprendizaje en la sociedad.

Las redes sociales forman parte activa de nuestras vidas desde unos años, han cambiado nuestra percepción del mundo e incluso nuestro modo de actuar en muchos aspectos, desde la forma que nos relaciones con otras personas.

\section{Referencias Bibliográficas}

Adell, J. (1997). Tendencias en educación en la sociedad de las tecnologías. Colombia.

Aguades, Ramírez. (2008) La importancia de las redes sociales en el desarrollo de competencias de la ciudadanía. págs. 167.

BALLESTA, J. (2000). Los medios de comunicación en la sociedad actual. España: Universidad de Murcia.

Barabasi A. L. (2002).Linked:The New Science of Networks, Cambridge, MA, Perseus Publishing.

Body, A., \& Allison, N. (2007). Social network sites: definition, history, and scholarship. España.

Dueñas, Comino. (2008) Educación y Redes Sociales. Los posibles caminos en la inserción y permanencia de sujetos vulnerables en micro-emprendimientos agrarios. México. págs. 200.

Esteruelas, C. (2012). La publicidad interactiva en España: Redes Sociales. España:Revista internacional de comunicación.

Gross, R. y Acquisti, A. (2005). Information Revelation and Privacy in Online Social Networks. Presentado en Workshop on Privacy in the Electronic Society. Alexandria: ACM Press. 
Hoyos, A. (2007) Influencia de las redes sociales en la educación superior. España: Anaya. . ISBN: 978-84-945243-2-5

Lozano, J. O. (2014). Las redes Sociales . Argentina: Mc Graw Hill.

Martínez-Guerrero, C. A. (2018). Uso de redes sociales en las revistas. Ciencias de la informacion, 21.

Pineda, M. (2009). Desafíos actuales de la sociedad del conocimiento para la inclusión digital en América Latina. Disertaciones, 2(1), 10-25. Recuperado de http://revistas.urosario.edu.co/index.php/disertaciones/article/view/3965/2923

Rodríguez, Cárdenas. (2008). Las redes sociales y la protección de datos hoy. Anuario Facultad de Derecho. España. págs..338.

Uribe, F. (2018). La importancia del apoyo social en el bienestar de los adolescentes. Psychologia, 4(2), p.2.

Zamora, J. (2004). Medios de comunicación, información, espectáculo, manipulación. Murcia. Editorial. Verbo Divino. Págs. 289. ISBN: 84-8169-639-0 


\section{Para citar el artículo indexado.}

Velasteguí E. (2017). Influencia de las Redes Sociales como herramienta de interacción en la educación. Revista electrónica Explorador Digital 2(1), 5-21. Recuperado desde: http://cienciadigital.org/revistacienciadigital2/index.php/exploradordigital/article/view/323/7 35

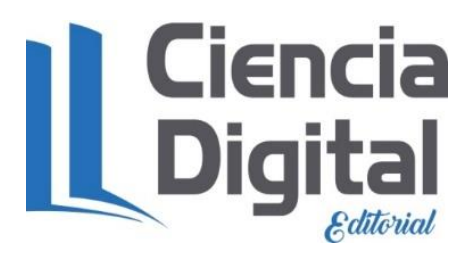

El artículo que se publica es de exclusiva responsabilidad de los autores y no necesariamente reflejan el pensamiento de la Revista Explorador Digital.

El articulo queda en propiedad de la revista y, por tanto, su publicación parcial y/o total en otro medio tiene que ser autorizado por el director o editor de la Revista Explorador Digital.
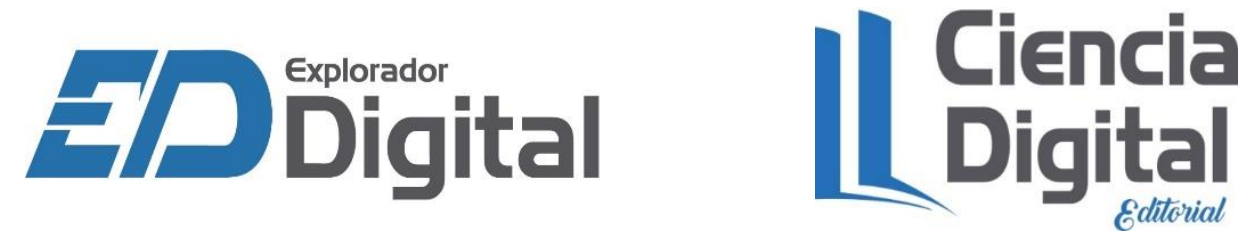